\title{
8. Survey participants
}

$\operatorname{AUTHOR}(\mathrm{S})$ :

OHGAKI, SHUN-ICHI; KOMEMOTO, KEN-ICHI; FUNAYAMA, NOBUTAKA

\section{CITATION:}

OHGAKI, SHUN-ICHI ...[et al]. 8. Survey participants. Publications of the Seto Marine Biological Laboratory. Special Publication Series 2011, 11: 189-190

\section{ISSUE DATE:}

2011

URL:

http://hdl.handle.net/2433/159492

RIGHT: 
8. Survey participants

Our survey team included staff members and research students of the Seto Marine Biological Laboratory of Kyoto University, students of Kyoto University and Nara Women's College, and local biologists from Wakayama Prefecture. A total of 82 personnel participated in the survey with a cumulative number of 237 personnel for the 26 censuses from 1985 to 2005 . The participants who were responsible for the identification of mollusk species are listed below in alphabetical order of their surnames.

1985: Makoto Kato, Takeharu Kosuge, Toshiaki Kuribayashi, Katsuki Nakai, Shun-ichi

Ohgaki, Ken Ohsuga, Koichi Takenouchi, Torao Yamamoto

1986: Makoto Kato, Takeharu Kosuge, Katsuki Nakai, Taisei Nakayama, Shun-ichi

Ohgaki, Koichi Takenouchi, Yasunori Uetani

1987: Takeshi Hashimoto, Yohei Iguchi, Makoto Kato, Katsuki Nakai, Taisei Nakayama,

Koichi Takenouchi, Yoshinobu Tanigawa, Yasunori Uetani, Shigefumi Yano

1988: Takeshi Hashimoto, Yohei Iguchi, Makoto Kato, Taisei Nakayama, Shun-ichi

Ohgaki, Koichi Takenouchi, Yoshinobu Tanigawa, Yasunori Uetani, Shigefumi Yano 1989: Takeshi Hashimoto, Yohei Iguchi, Makoto Kato, Taiji Kurozumi, Katsuki Nakai,

Koichi Takenouchi, Yoshinobu Tanigawa

1990: Takeshi Hashimoto, Shu-nichi Ohgaki, Ken Ohsuga, Koichi Takenouchi, Yoshinobu

Tanigawa, Shigefumi Yano

1991: Naohiro Aizawa, Takeshi Hashimoto, Yohei Iguchi, Makoto Kato, Katsuki Nakai,

Shun-ichi Ohgaki, Ken Ohsuga, Wataru Ohtsuka, Koichi Takenouchi, Yoshinobu

Tanigawa

1992: Naohiro Aizawa, Takeshi Hashimoto, Katsuki Nakai, Shun-ichi Ohgaki, Wataru

Ohtsuka, Koichi Takenouchi, Yoshinobu Tanigawa

1993: Naohiro Aizawa, Takeshi Hashimoto, Yasunori Kano, Taisei Nakayama, Shun-ichi

Ohgaki, Koichi Takenouchi, Shigeyuki Yamato

1994: Naohiro Aizawa, Takeshi Hashimoto, Yasunori Kano, Katsuki Nakai, Taisei 
Nakayama, Shunichi Ohgaki, Koichi Takenouchi, Yoshinobu Tanigawa 1995: Naohiro Aizawa, Takeshi Hashimoto, Yohei Iguchi, Katsuki Nakai, Taisei Nakayama, Shun-ichi Ohgaki, Wataru Ohtsuka, Koichi Takenouchi, Yoshinobu Tanigawa 1996: Yohei Iguchi, Taisei Nakayama, Shun-ichi Ohgaki, Wataru Ohtsuka, Koichi

Takenouchi, Yoshinobu Tanigawa, Tomoko Yamamoto 1997: So Ishida, Taisei Nakayama, Shun-ichi Ohgaki, Michiko Sato, Koichi Takenouchi, Yasunori Uetani, Shigeyuki Yamato 1998: Katsuki Nakai, Taisei Nakayama, Shun-ichi Ohgaki 1999: Nobutaka Funayama, So Ishida, Kenichi Komemoto, Shun-ichi Ohgaki, Wataru Ohtsuka, Shigeyuki Yamato 2000: Nobutaka Funayama, Ken-ichi Komemoto, Takeshi Kurihara, Shun-ichi Ohgaki, Michiko Sato, Hidetomo Tanase, Shigeyuki Yamato 2001: Nobutaka Funayama, So Ishida, Shun-ichi Ohgaki, Michiko Sato, Hidetomo Tanase, Shigeyuki Yamato 2002: Nobutaka Funayama, Nobutaka Funayama, Ken-ichi Komemoto, Shun-ichi Ohgaki, Michiko Sato, Hidetomo Tanase 2003: Shun-ichi Ohgaki 2004: Nobutaka Funayama, Ken-ichi Komemoto, Shun-ichi Ohgaki, Hidetomo Tanase, Shigeyuki Yamato 2005: Nobutaka Funayama, Ken-ichi Komemoto, Shun-ichi Ohgaki, Shigeyuki Yamato 2006: Nobutaka Funayama, Ken-ichi Komemoto, Shun-ichi Ohgaki 2007: Nobutaka Funayama, Ken-ichi Komemoto, Shun-ichi Ohgaki 2008: Nobutaka Funayama, Shun-ichi Ohgaki 2009: Ken-ichi Komemoto, Shun-ichi Ohgaki 2010: Nobutaka Funayama, Ken-ichi Komemoto, Shun-ichi Ohgaki 